\title{
Machine Learning in Neuroimaging: A New Approach to Understand Acupuncture for Neuroplasticity
}

\author{
Tao Yin, ${ }^{1,2}$ Peihong Ma, ${ }^{1,2}$ Zilei Tian, ${ }^{1,2}$ Kunnan Xie, ${ }^{1,2}$ Zhaoxuan He, ${ }^{1,2}$ Ruirui Sun, ${ }^{1,2}$ \\ and Fang Zeng $\mathbb{D}^{1,2,3}$ \\ ${ }^{1}$ Acupuncture and Tuina School/The Third Teaching Hospital, Chengdu University of Traditional Chinese Medicine, Chengdu, \\ Sichuan, China \\ ${ }^{2}$ Acupuncture \& Brain Science Research Center, Chengdu University of Traditional Chinese Medicine, Chengdu, Sichuan, China \\ ${ }^{3}$ Key Laboratory of Sichuan Province for Acupuncture and Chronobiology, Chengdu University of Traditional Chinese Medicine, \\ Chengdu, Sichuan, China
}

Correspondence should be addressed to Fang Zeng; zeng_fang@126.com

Received 25 June 2020; Revised 2 August 2020; Accepted 8 August 2020; Published 24 August 2020

Academic Editor: Jing-Wen Yang

Copyright ( 2020 Tao Yin et al. This is an open access article distributed under the Creative Commons Attribution License, which permits unrestricted use, distribution, and reproduction in any medium, provided the original work is properly cited.

\begin{abstract}
The effects of acupuncture facilitating neural plasticity for treating diseases have been identified by clinical and experimental studies. In the last two decades, the application of neuroimaging techniques in acupuncture research provided visualized evidence for acupuncture promoting neuroplasticity. Recently, the integration of machine learning (ML) and neuroimaging techniques becomes a focus in neuroscience and brings a new and promising approach to understand the facilitation of acupuncture on neuroplasticity at the individual level. This review is aimed at providing an overview of this rapidly growing field by introducing the commonly used ML algorithms in neuroimaging studies briefly and analyzing the characteristics of the acupuncture studies based on ML and neuroimaging, so as to provide references for future research.
\end{abstract}

\section{Introduction}

Neuroplasticity usually refers to brain plasticity, which means the ability of the brain to modify its organization to the altered demands and environments [1,2]. The cumulative evidence from both animal and human studies demonstrated that the adult mammalian brain was plastic and could be remodeled by the environmental input continuously [3-5]. The long-term noxious stimulus, such as pain and depression, as well as regular exogenous interventions can reorganize the structure and function of the brain [6-9]. As the most widely used complementary therapy, acupuncture is considered to treat diseases via facilitating neural plasticity from multiple pathways, such as promoting endogenous neurogenesis, modulating synaptic plasticity, and regulating the secretion of neurotrophins and neurotransmitters, so as to affect the structural and functional plasticity of the brain [10-13].
In the past two decades, studies on acupuncture promoting brain plasticity were greatly enhanced with the development of neuroimaging techniques. Several studies focused on investigating acupuncture-induced brain structural and functional plasticity by magnetic resonance imaging (MRI), positron emission tomography (PET), and other neuroimaging methods $[14,15]$. People found that acupuncture could modulate the brain functional activities, shape the gray matter structure, and remodel the white matter fiber connection [16-18] and that the modulation of acupuncture on neuroplasticity varied with the different acupuncture modalities and different acupoint stimulations $[19,20]$. For instance, our previous study [21] found that acupuncture could positively modulate the functional activity of the rostral ventromedial medulla in patients with migraine and that the neural plasticity elicited by puncturing at real acupoints was more pronounced than sham acupoints. 


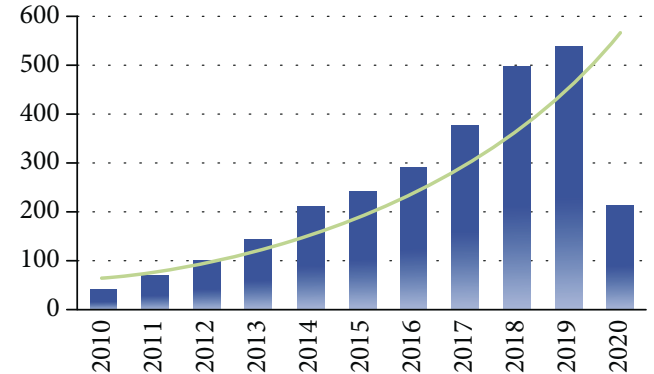

FIGURE 1: Numbers of publication on neuroimaging and machine learning in the last decade (from January 1, 2010, to June 1, 2020). The data was obtained by searching at the PubMed database with the items (Neuroimaging) AND (Machine Learning).

Currently, most neuroimaging findings of acupuncture facilitating neuroplasticity were obtained by the standard univariate analysis. It means the results were only significant at the group level, which limited their clinical translation to a certain extent. So, it is of great value to investigate how acupuncture promotes neuroplasticity and how the specific neuroplasticity affects the responses to acupuncture from the individual level. The application of multivariate pattern analysis (MVPA) and machine learning (ML) in neuroimaging studies provides an attractive method to this issue [22]. Since 2010, over 2200 studies focusing on ML in neuroimaging have been published in PubMed (pubmed. ncbi.nlm.nih.gov), and the number of these studies is increasing by $37 \%$ per year (Figure 1). With the ML algorithms and the neuroimaging features, researchers established the diagnostic and prognostic models of diseases. The interpretation of these models complemented the deficiencies of univariate analysis. They can not only assist in diagnosing diseases and in predicting individuals' responses to intervention but also provide novel insights for understanding brain plasticity. For example, Min et al. [23] found that schizophrenics who were sensitive to electroconvulsive therapy (ECT responders) had significantly higher whole-brain transfer entropy than the ECT nonresponders and that the value of whole-brain transfer entropy could be used as a reliable and plausible neuroimaging biomarker for random forest (RF) classifier to identify the ECT responders from the nonresponders. In another study, applying the baseline gray matter volume (GMV) of the subgenual cingulate cortex as a feature, Redlich and colleagues [24] successfully predicted the continued improvement of depression symptoms in patients with major depressive disorder following ECT. Simultaneously, integrating $\mathrm{ML}$ and neuroimaging technologies to investigate the facilitation of acupuncture on brain plasticity and using specific brain plasticity to predict acupuncture efficacy which can promote precision treatment have been a new focus in acupuncture research.

Therefore, we conducted this review by introducing the most widely used ML algorithms in neuroimaging studies briefly and analyzing these applications in the fieldof acupuncture promoting neural plasticity, aiming to provide an overview of this rapidly growing field and new approaches in future research.

\section{Overview of Machine Learning in Neuroimaging}

ML is a subfield of artificial intelligence which is aimed at investigating how computers can improve decisions and predictions based on data and ongoing experience [25, 26]. According to the criteria whether the training data is given a label or not, ML is divided into supervised learning, unsupervised learning, and semisupervised learning [27]. The unsupervised learning and semisupervised learning are generally applied for data reduction and feature selection [28], whereas the supervised learning is mainly used to construct the classification or regression models, which can learn the mappings between the input features and labels, to make individual-level estimations for the previously unseen data. The supervised learning includes many types, of which the most commonly used in neuroimaging research include support vector machine (SVM), decision tree (DT), RF, and artificial neural network (ANN) [29].

2.1. Support Vector Machine. The SVM is so far the most popular supervised learning algorithm in neuroimaging studies and is widely utilized in classification and prediction [30-33]. The principle of SVM is constructing a separating hyperplane that classifies all inputs, and the goal is searching for the optimal separating hyperplane that maximizes the margin between the hyperplane and the support vectors [34]. With different kernel functions, the distinct separating hyperplanes in different dimensions were constructed to perform the classification or prediction analysis. Among the different kinds of kernel functions in SVM models, the linear kernel and Gaussian kernel are most frequently used in neuroimaging studies [35-37]. The linear SVM is designed to solve the linear separating problems, while the RBF SVM is used primarily to seek nonlinear separating boundaries in the high-dimensional space.

2.2. Decision Tree and Random Forest. DT is the rooted directed tree that predicts the output based on a sequence of splits in the input feature space. The nodes split at each step by optimizing a metric, which indicates the consistency between the estimates and truth values. When the node has no subordinate to split, the traversal of this tree generates the target outcome prediction. As a typical classification algorithm with high interpretability, DT is applied predominantly for classification and disease diagnosis in neuroimaging studies $[38,39]$.

$\mathrm{RF}$ is generally the ensembles of DTs [40]. The principle of RF is consolidating multiple and diverse DTs together, and the final prediction outcome of RF is determined by the votes of each DT in the forest. As an integrated algorithm, RF can potentially yield much better prediction performance than learning with a single DT [41].

2.3. Artificial Neural Network. The concept of ANN is derived from the biological neural network. Similar to the synaptic connection in the brain, an ANN is composed of several layers of interconnected artificial neurons that make up the input layer, hidden layer, and output layer. As an 

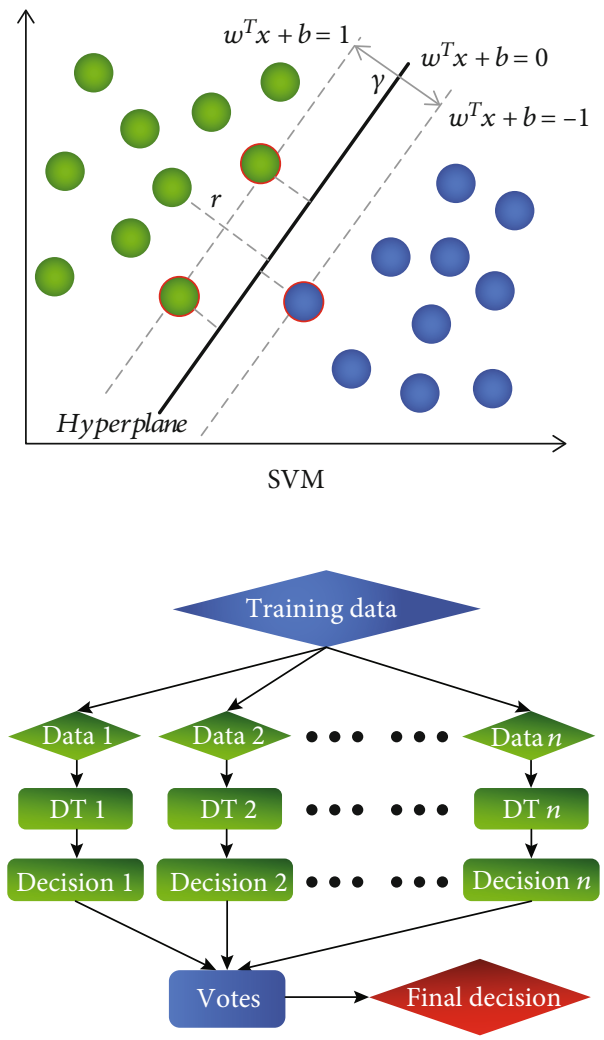

RF
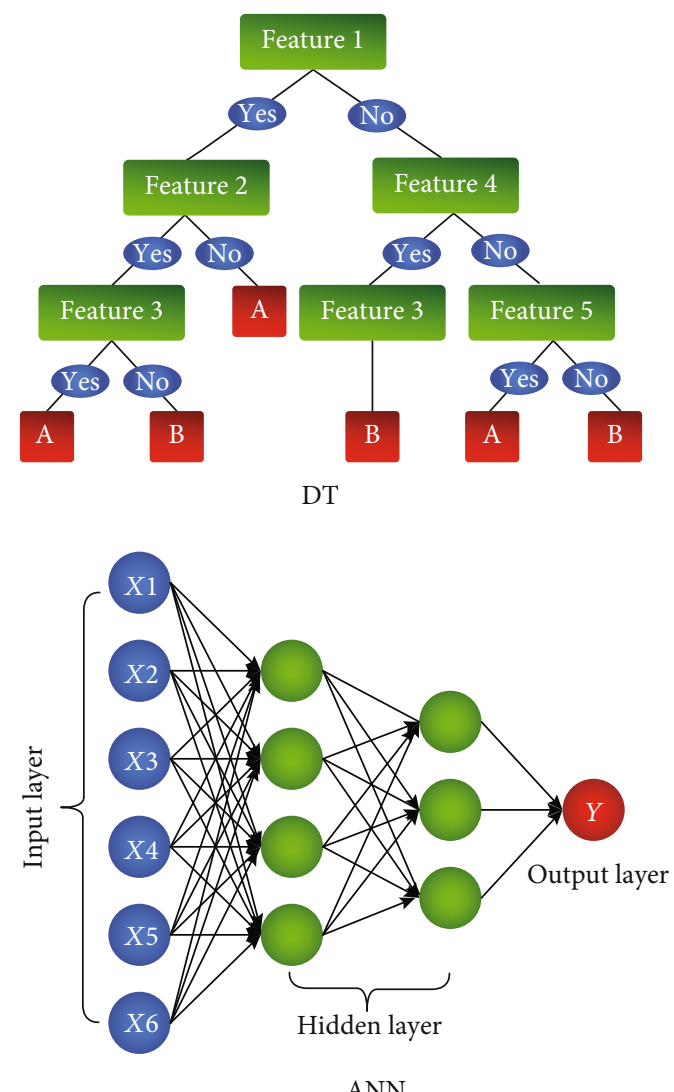

FIgURE 2: Diagrams of the commonly used machine learning algorithms in neuroimaging studies. SVM: support vector machine; DT: decision tree; RF: random forest; ANN: artificial neural network.

ultracomplex ML algorithm, ANN establishes the computational units of multiple layers by simulating signal transmission and learning the architecture of synapse [42]. Due to the flexibility of its structure, ANN has the ability to fit arbitrarily complex functions given sufficient annotated data [27]. Traditionally, the utilization of ANN is extremely limited in neuroimaging for the small training samples, while in recent years, with benefit from the open-access of the large-scale neuroimaging data repositories, the application of ANN is accelerating and has great potential to become one of the most efficient algorithms in neuroimaging studies $[43,44]$.

The diagrams of the above algorithms are summarized in Figure 2 .

\section{Application of Neuroimaging and Machine Learning in Acupuncture Promoting Neuroplasticity}

In this review, we focused on the application of neuroimaging and ML in acupuncture promoting neuroplasticity. For a comprehensive summary of the field, we systematically searched papers in PubMed (pubmed.ncbi.nlm.nih.gov), Web of Science (https://www.webofknowledge.com), EBSCO (search.ebscohost.com), and CNKI (https://www.cnki.net) databases. According to the established inclusion and exclusion criteria, a total of ten studies were finally included
[45-54]. The details of data acquisition and literature selection process can be found in the supplementary materials (available here).

These ten studies were published from 2008 to 2020 . Generally, for participant selection, these studies were performed on healthy subjects [45-47, 49, 54], patients with migraine $[48,50,51]$, patients with chronic low back pain [53], and patients with functional dyspepsia [52], respectively. The sample size of these studies ranged from 12 to 94 , and the average sample size of healthy subjects was 28 . Except for one study that enrolled participants with a wide age span [54], these studies mainly included participants aged 20-45 years. For acupuncture intervention, nine studies [45-53] applied manual acupuncture, and one study [54] selected the electroacupuncture as the intervention method. For scan design, six studies $[45-49,54]$ applied the on-off block design to detect the real-time effects of acupuncture on functional brain plasticity. For imaging parameters, seven studies [45-47, 50-53] employed MRI to acquire neuroimaging data and applied the blood oxygenation level-dependent (BOLD) signal [45-47], functional connectivity [52, 53], GMV [51], and diffusion measures of white matter fibers [50] to reflect the structural and functional patterns of the brain. For machine learning parameters, eight studies [45-52] were aimed at solving the problems of pattern classification, and the other two studies $[53,54]$ were designed to predict pain relief following acupuncture treatment. 
SVM, especially the linear SVM, was the most used ML algorithm in classification [45-52], whereas support vector regression [53] and fuzzy neural network [54] were applied in predictions. Three studies $[49,53,54]$ exploited a hypothesis-based approach and selected the features of interest as inputs, and the other three studies $[45,50,51]$ integrated multiple methods to find the optimal inputted features. Cross-validation, particularly the leave-one-outcross-validation, was the popular validation strategy [45, 47-53], and only one study used independent samples as the validation set in these ten studies [54].

The detailed characteristics of these included studies were displayed in Table 1.

\subsection{Concerns of Studies on Acupuncture Promoting} Neuroplasticity. According to aims and design, these studies can be divided into three types. Among them, three studies [45-47] focused on the acupoint specificity, two studies [48, 49] were concerned with the differences and similarities of different acupuncture manipulations, and five studies [5054] paid their attention to the prediction of acupuncture efficacy.

3.1.1. The Acupoint Specificity. Acupoint specificity refers that acupoints have different therapeutic effects and biophysical characteristics compared to sham acupoints and that different acupoints have relatively different therapeutic effects and biophysical characteristics [55]. In this review, three studies [45-47] focused on the acupoint specificity. One study was aimed at exploring the differences in real-time brain functional plasticity elicited by a verum acupoint and a sham acupoint. The other two studies compared the differences between different acupoints (GB40 vs. KI3 [46] and HT7 vs. PC6 [47]). These three studies had similar experimental designs, including focusing on the different points in the same nerve segments, using the multiple on-off block design, choosing the BOLD signal as features, and adopting the linear SVM algorithm to build models. For example, Li et al. [45] applied MVPA and searchlight method to decode spatial discrimination of acupuncture stimulation at GB37 and a nearby sham acupoint. The results indicated that the occipital cortex, limbic-cerebellar areas, and somatosensory cortex were the main regions with higher classification accuracy in the discrimination of the verum and sham acupoint stimulation. These studies indicated that acupuncture stimulation at different points induced distinct real-time brain functional plasticity in different regions and that MVPA could be used to investigate the real-time neuroplasticity from the individual level. Interestingly, these three studies utilized the general linear model (GLM) analysis to verify the findings obtained in MVPA, while every GLM analysis showed that the different points caused similar BOLD signal changes. It suggested that the conventional univariate analysis might not be sensitive enough to detect the neural plasticity evoked by different acupoint stimulation. This is consistent with the opinion that multivariate analysis was more sensitive than univariate analysis in neuroimaging studies [56].
Acupoint specificity is not only the core of acupuncture theory and the base of clinical practice but also the focus of acupuncture-neuroimaging research [57-59]. Our previous review [15] indicated that more than $1 / 3$ acupunctureneuroimaging studies focused on acupoint specificity and these studies mainly concentrated on the differences of verum acupoints and sham acupoints. From 1995 to 2016, 79 original neuroimaging articles on acupoint specificity were published in PubMed, and 53 articles focused on the difference between the verum acupoints and the sham acupoints [19]. Given the importance of acupoint specificity in acupuncture theory and clinical practice and the extensiveness in neuroimaging research, we hold that acupoint specificity is bound to become a hot spot in future ML and neuroimaging studies.

3.1.2. Acupuncture Manipulation. Two [48, 49] of the ten studies centered on the differences in brain functional plasticity caused by the different acupuncture manipulations. In one study [48], linear SVM was applied to classify the baseline and post acupuncture blood perfusion patterns in both verum and sham acupuncture groups. The results illustrated that the SVM classifier performed better when the training data was extracted from the verum acupuncture group. Moreover, the temporal lobe and cerebellum contributed important information for the discrimination in the verum acupuncture group. Another study [49] proposed a classification framework based on multiple ML algorithms for the two traditional acupuncture manipulations: the twirling-rotating manipulation and lifting-thrusting manipulation. The results demonstrated that with all the six graph theory properties as inputs, the SVM classifier got the highest accuracy of $92.14 \%$. Moreover, the post hoc analysis also found the significant between-group differences of these six graph theory measures between two manipulations.

Acupuncture manipulation is the key in acupuncture clinical practice and significantly affects acupuncture efficacy [60]. In more than 2000 years of development, acupuncture has formed a rich variety of modalities and manipulation skills. The differences between acupuncture and moxibustion, electroacupuncture and manual acupuncture, acupuncture with deqi and acupuncture without deqi, and the reinforcing manipulation and reducing manipulation are always the key of clinical and experimental research in the acupuncture field and could be the research direction in future MVPA studies.

3.1.3. Prediction of Acupuncture Efficacy. The integration of ML and neuroimaging features has been extensively employed in predicting the clinical efficacy of drugs or other interventions [33,61]. In this review, five studies focused on acupuncture efficacy prediction [50-54]. Among them, three studies [50-52] adopted the classification algorithms to predict patients' responses to acupuncture treatment. For example, Liu et al. [50] utilized the diffusion measures of the medial prefrontal cortex- (mPFC-) amygdala fiber as inputs and established a linear SVM classifier to predict the response of migraine patients to the 8-week sham acupuncture treatment. The result showed that when using each 


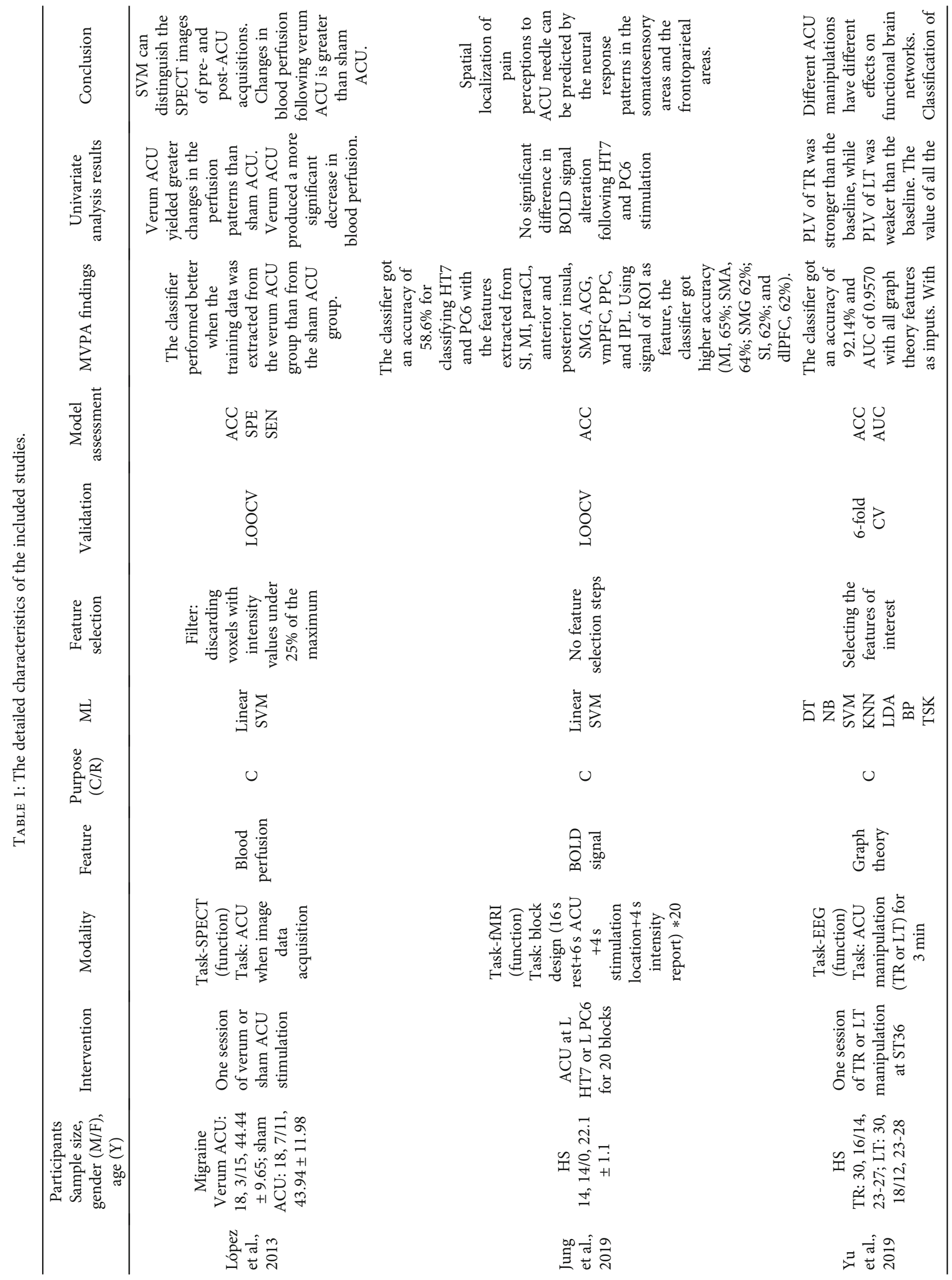




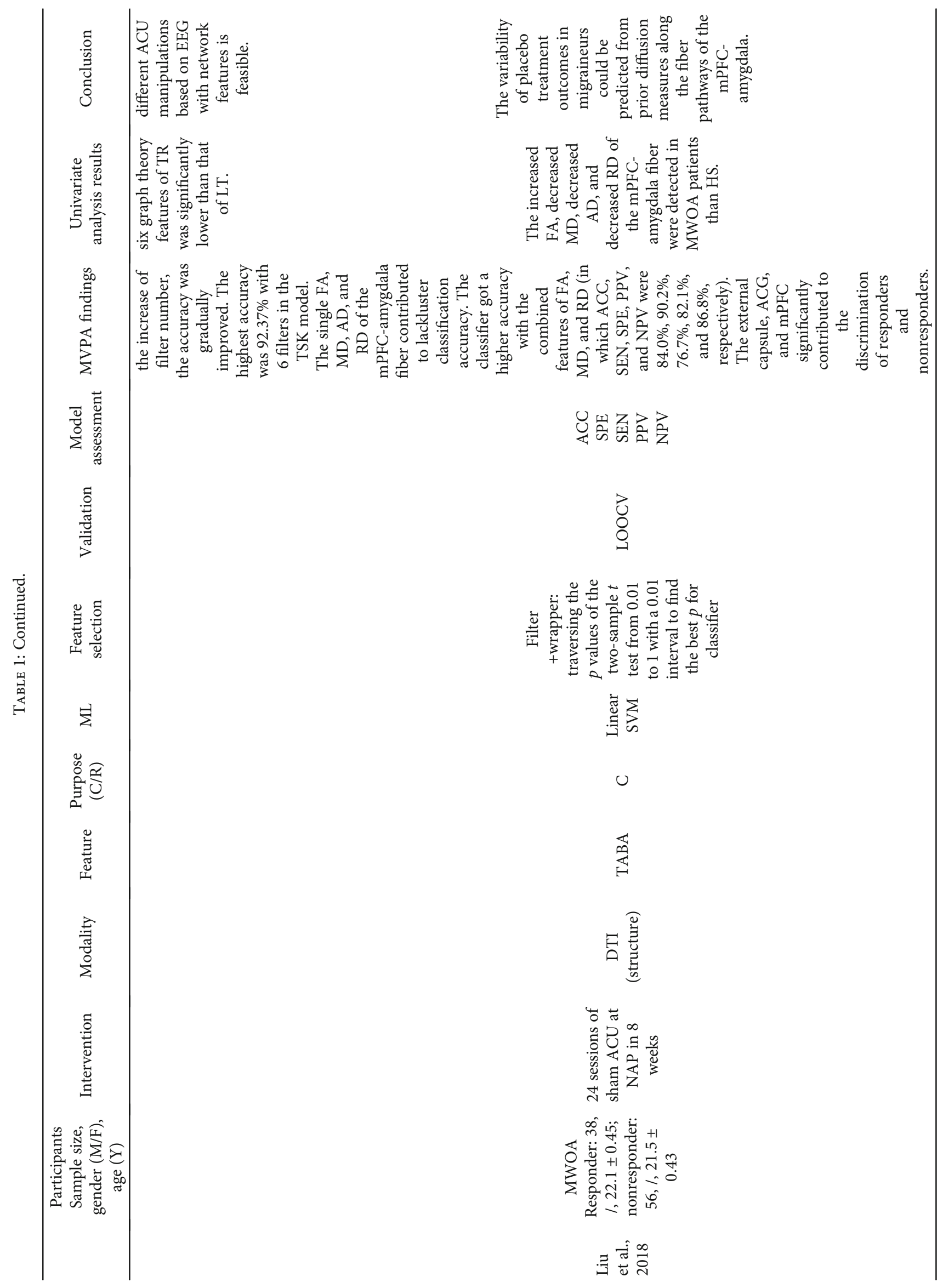




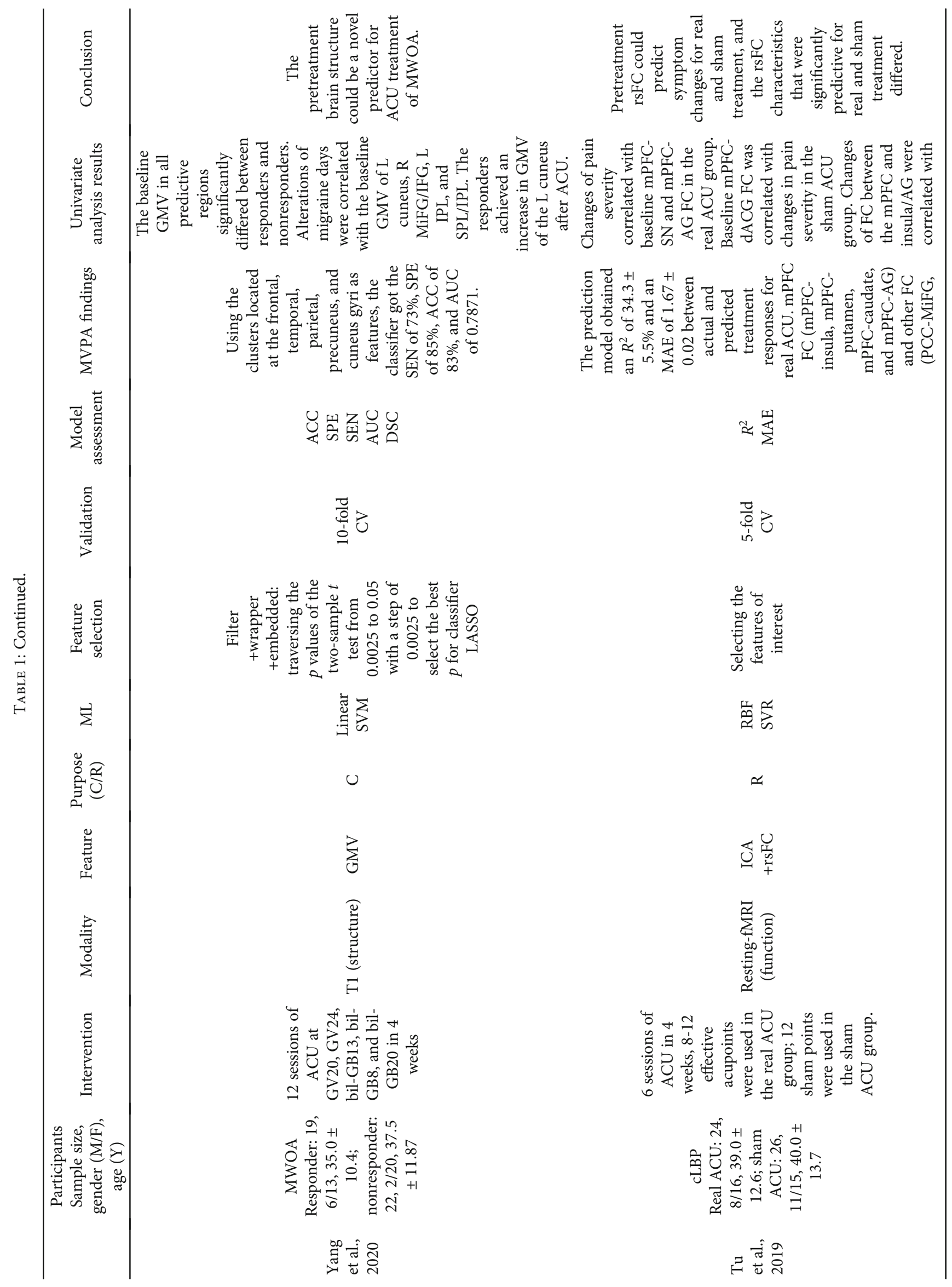




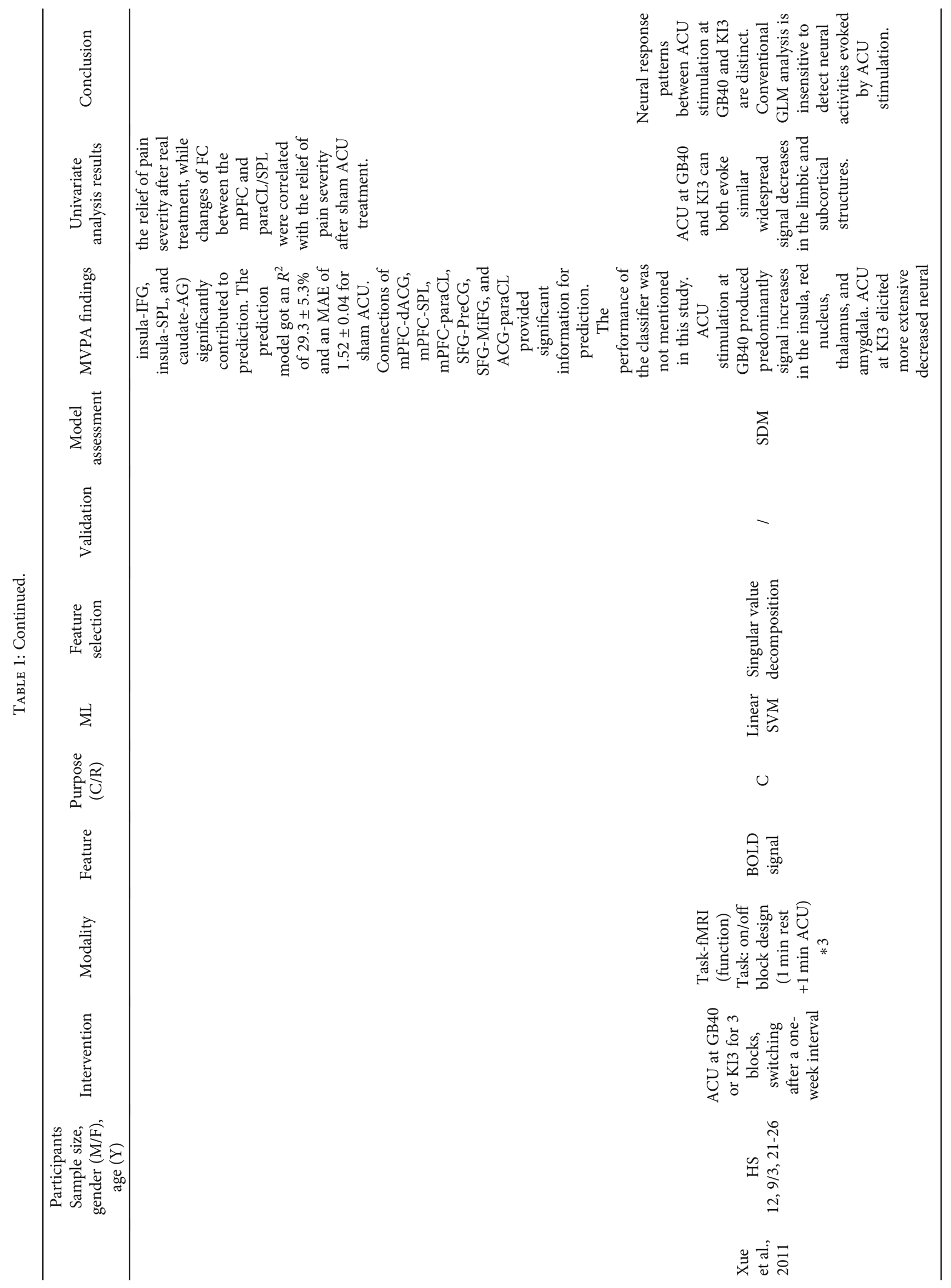




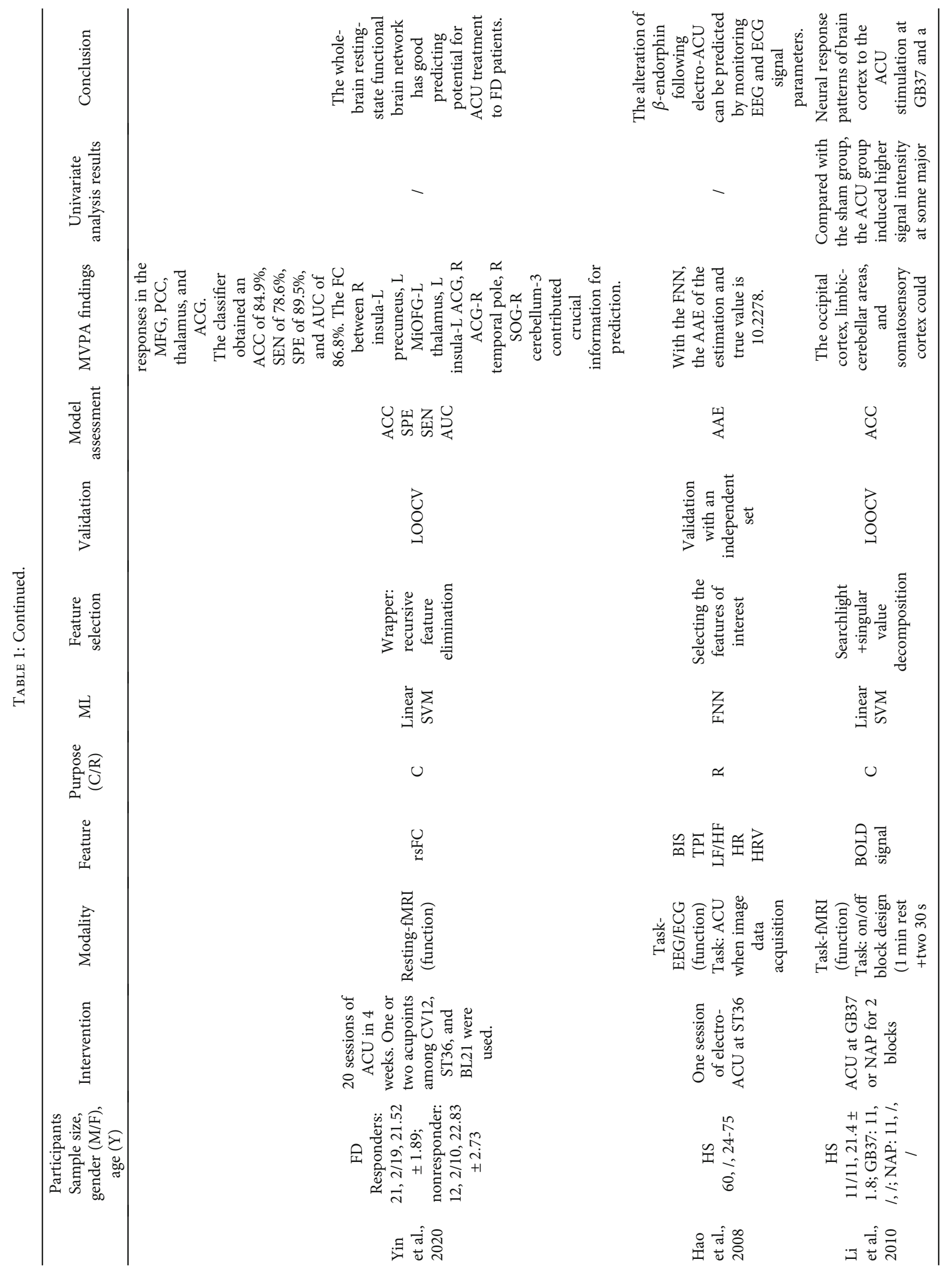




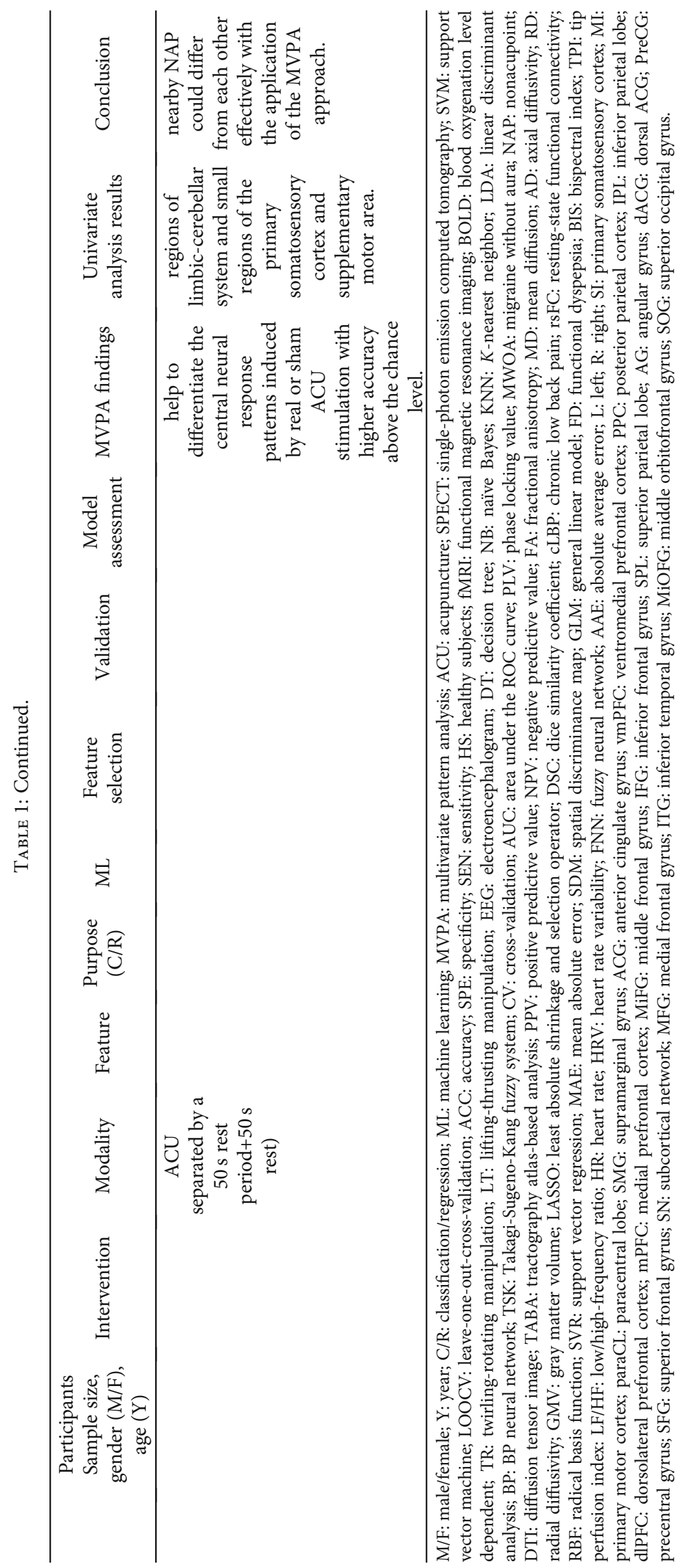


single diffusion measure as input, the accuracy of the classifier is lackluster, whereas when multiple measures were applied the classifier could accurately discriminate responders from nonresponders with an accuracy of $84.0 \%$. Moreover, the most discriminative white matter plasticity features that contributed to the classification were located in the external capsule, anterior cingulate gyrus, and $\mathrm{mPFC}$. The other two studies $[53,54]$ constructed the regression models to predict the continuous improvement in symptoms after acupuncture treatment. For example, Tu et al. [53] used the features of interest as inputs to predict pain relief in patients with cLBP following 8 -week verum or sham acupuncture treatment. The results showed that multiple functional connections involving mPFC could provide vital information for predicting the improvement of symptoms after both verum and sham acupuncture treatment.

These five studies on acupuncture efficacy prediction demonstrated that the specific neuroplasticity features including morphology of gray matter and white matter and cerebral functional activity patterns contained vital information for predicting the response of patients to acupuncture stimulation. The integration of ML and neuroimaging provides a new and promising approach for investigating mechanisms of acupuncture efficacy at the individual level, which has great potential for clinical translation and will be the important growth pole in acupuncture research.

In addition to the three aspects described above, there are still some other concerns that should be focused in future neuroimaging-based ML studies, for example, investigating the influences of acupuncture with different acupoint combination or different stimulation intensity on neural plasticity and predicting clinical efficacy of acupuncture with the neuroimaging features acquired under acupuncture stimulation.

\subsection{Design of Machine Learning in Studies on Acupuncture} Promoting Neuroplasticity. The application of neuroimaging techniques in acupuncture mechanism has produced remarkable advance $[57,62,63]$ and developed a series of proven execution specifications $[14,19,64]$. In contrast, the integration of $\mathrm{ML}$ and neuroimaging in acupuncture research is still in its early stage, which inevitably brings many challenges but also the future directions.

3.2.1. Sample Size. Due to difficulties in data acquisition, the sample size of neuroimaging study is generally small $[65,66]$. By reviewing the studies which integrated ML and neuroimaging technologies to investigate neuropsychiatric disorders, Sakai and Yamada [29] found that $45.6 \%$ of the studies from 2014 to 2018 had a sample size of fewer than 100 cases. In our review, the sample size of the included studies ranged from 12 to 94 and six studies had a sample size of fewer than 50 cases. A small sample size exacerbates the possibility of adaptive models to learn noise, which leads to the high variability of estimates and overvaluation of prediction accuracy [67]. Simulation experiments showed that even when the sample size in the neuroimaging study reached 100 cases, the error bars were still around 10\% [68]. Only when the samples of the training set exceeded 200 cases did the prediction model's performance begin to plateau [69].
Therefore, when conducting an ML study to predict the efficacy of acupuncture based on the neuroimaging properties, a sample size of 200 or more cases should be guaranteed whenever possible.

3.2.2. The Appropriateness of Feature Selection. Considering that there are generally more features than samples in neuroimaging data, it is beneficial to take appropriate manners to eliminate the redundant features and reduce the dimension of data. The ten studies included in this review indicated that when using a single feature as input, the accuracy of the classifier is lackluster, whereas when multiple neuroimaging features applied, the accuracy of the model was significantly improved $[49,50]$. This finding suggested that the properties of neuroplasticity that influenced the efficacy of acupuncture were multidimensional and complex. Moreover, another interesting finding was that both GMV and diffusion measures of white matter fiber could accurately discriminate between acupuncture-sensitive and acupuncture-insensitive migraine patients $[50,51]$. Does it mean that the prediction model achieves better performance to discriminate the acupuncture responders and acupuncture nonresponders if both gray matter and white matter features are applied as inputs? In fact, the previous studies have illustrated that using multimodal rather than single-modal neuroimaging features as inputs can induce higher classification accuracy and better prediction performance $[70,71]$. Therefore, future studies could attempt to use multimodal neuroimaging features as inputs to further explore the multidimensional features that predict the efficacy of acupuncture accurately.

3.2.3. The Representativeness of Training Data. The current ML studies generally favor seeking homogeneous subjects to establish classification and prediction models [72-74]. It reduces the underfitting of the model caused by data heterogeneity, but severely limits the generalizability of the model to the real-world data [75]. The requirements for the representativeness of training data depend on the purpose of the study. For example, when a study is aimed at investigating the effects of different acupuncture manipulations on brain plasticity, the participants should be the homogeneous individuals from the same site. However, if the study is aimed at creating a generalizable model to predict the clinical efficacy of acupuncture, the participants should be enrolled from multiple centers to represent the heterogeneous population in real life.

3.2.4. The Validity of Labels. The goal of ML is establishing mappings between training data and labels and then use the mappings as benchmarks for predicting the labels of the unseen data. Similar to other ML studies [76-78], the majority of current studies on acupuncture efficacy prediction use the subjective symptoms as the labels. These labels obtained with self-evaluated symptoms are subject to individual cognitive bias and have a high degree of variability. The heterogeneity yielded by subjective labels may hamper ML algorithms to discover optimal neuroimaging biomarkers and establish accurate mappings between data and labels. Therefore, applying objective biological markers as labels to establish 
an objective-to-objective mapping between features and labels should be taken into consideration in future studies to reduce the influence of subjective factors on model reliability.

\section{Conclusion}

In summary, we provided an overview of the literature on the application of ML and neuroimaging in acupuncture promoting neural plasticity. Studies published so far have preliminarily demonstrated at the individual level that different acupoint stimulation and different acupuncture manipulations had significantly different real-time modulatory effects on functional brain plasticity and that the specific structural and functional neuroplasticity features at baseline could accurately predict the improvement of symptoms following acupuncture treatment. Although this research field is currently in its early stage and faces many challenges, we still believe that integrating $\mathrm{ML}$ and neuroimaging techniques will be a promising approach to understand the facilitation of acupuncture on neuroplasticity in the future.

\section{Data Availability}

There is no original data in this review.

\section{Conflicts of Interest}

The authors declare that they have no competing interests.

\section{Authors' Contributions}

Tao Yin, Peihong Ma, and Zilei Tian contributed equally to this work.

\section{Acknowledgments}

This work was supported by the National Key R\&D Program of China (No. 2018YFC1704600 and No. 2018YFC1704605), the National Natural Science Foundation of China (No. 81973960), and the Sichuan Science and Technology Program (No. 2020JDRC0105).

\section{Supplementary Materials}

The details of data acquisition and literature selection process. (Supplementary Materials)

\section{References}

[1] K. Hötting and B. Röder, "Beneficial effects of physical exercise on neuroplasticity and cognition," Neuroscience \& Biobehavioral Reviews, vol. 37, no. 9, pp. 2243-2257, 2013.

[2] H. van Praag, G. Kempermann, and F. H. Gage, "Neural consequences of environmental enrichment," Nature Reviews Neuroscience, vol. 1, no. 3, pp. 191-198, 2000.

[3] A. Pascual-Leone, A. Amedi, F. Fregni, and L. B. Merabet, "The plastic human brain cortex," Annual Review of Neuroscience, vol. 28, no. 1, pp. 377-401, 2005.
[4] L. Mandolesi, F. Gelfo, L. Serra et al., "Environmental factors promoting neural plasticity: insights from animal and human studies," Neural Plasticity, vol. 2017, Article ID 7219461, 10 pages, 2017.

[5] C. H. Zhang, Z. Z. Ma, B. B. Huo et al., "Diffusional plasticity induced by electroacupuncture intervention in rat model of peripheral nerve injury," Journal of Clinical Neuroscience, vol. 69, pp. 250-256, 2019.

[6] R. Kuner and H. Flor, "Structural plasticity and reorganisation in chronic pain," Nature Reviews Neuroscience, vol. 18, no. 1, pp. 20-30, 2016.

[7] E. Duzel, H. van Praag, and M. Sendtner, "Can physical exercise in old age improve memory and hippocampal function?," Brain, vol. 139, no. 3, pp. 662-673, 2016.

[8] H. Johansson, M. Hagströmer, W. J. A. Grooten, and E. Franzén, "Exercise-induced neuroplasticity in Parkinson's disease: a metasynthesis of the literature," Neural Plasticity, vol. 2020, Article ID 8961493, 15 pages, 2020.

[9] W. Liu, T. Ge, Y. Leng et al., "The role of neural plasticity in depression: from hippocampus to prefrontal cortex," Neural Plasticity, vol. 2017, Article ID 6871089, 11 pages, 2017.

[10] L. Y. Xiao, X. R. Wang, Y. Yang et al., "Applications of acupuncture therapy in modulating plasticity of central nervous system," Neuromodulation: Technology at the Neural Interface, vol. 21, no. 8, pp. 762-776, 2018.

[11] V. Protto, M. Soligo, M. E. de Stefano et al., "Electroacupuncture in rats normalizes the diabetes-induced alterations in the septo-hippocampal cholinergic system," Hippocampus, vol. 29, no. 10, pp. 891-904, 2019.

[12] H. C. Lai, Q. Y. Chang, and C. L. Hsieh, "Signal transduction pathways of acupuncture for treating some nervous system diseases," Evidence-based Complementary and Alternative Medicine, vol. 2019, Article ID 2909632, 37 pages, 2019.

[13] L. M. Chavez, S. S. Huang, I. MacDonald, J. G. Lin, Y. C. Lee, and Y. H. Chen, "Mechanisms of acupuncture therapy in ischemic stroke rehabilitation: a literature review of basic studies," International Journal of Molecular Sciences, vol. 18, no. 11, article 2270, 2017.

[14] Z. He, L. Hou, R. Sun et al., "The status of the acupuncture mechanism study based on PET/PET-CT technique: design and quality control," Evidence-based Complementary and Alternative Medicine, vol. 2019, Article ID 9062924, 8 pages, 2019.

[15] K. Qiu, M. Jing, R. Sun et al., "The status of the quality control in acupuncture-neuroimaging studies," Evidence-based Complementary and Alternative Medicine, vol. 2016, Article ID 3685785, 14 pages, 2016.

[16] Y. Zou, W. Tang, X. Li, M. Xu, and J. Li, “Acupuncture reversible effects on altered default mode network of chronic migraine accompanied with clinical symptom relief," Neural Plasticity, vol. 2019, Article ID 5047463, 10 pages, 2019.

[17] P. Wu, Y. M. Zhou, C. X. Liao et al., "Structural changes induced by acupuncture in the recovering brain after ischemic stroke," Evidence-based Complementary and Alternative Medicine, vol. 2018, Article ID 5179689, 8 pages, 2018.

[18] H. Kim, I. Mawla, J. Lee et al., "Reduced tactile acuity in chronic low back pain is linked with structural neuroplasticity in primary somatosensory cortex and is modulated by acupuncture therapy," NeuroImage, vol. 217, article 116899, 2020.

[19] K. Qiu, T. Yin, X. Hong et al., "Does the acupoint specificity exist? Evidence from functional neuroimaging studies," Current Medical Imaging, vol. 16, no. 6, pp. 629-638, 2020. 
[20] R. L. Cai, G. M. Shen, H. Wang, and Y. Y. Guan, "Brain functional connectivity network studies of acupuncture: a systematic review on resting-state fMRI," Journal of Integrative Medicine, vol. 16, no. 1, pp. 26-33, 2018.

[21] Z. Li, F. Zeng, T. Yin et al., "Acupuncture modulates the abnormal brainstem activity in migraine without aura patients," Neuro Image Clinical, vol. 15, pp. 367-375, 2017.

[22] J. V. Haxby, "Multivariate pattern analysis of fMRI: the early beginnings," NeuroImage, vol. 62, no. 2, pp. 852-855, 2012.

[23] B. Min, M. Kim, J. Lee et al., "Prediction of individual responses to electroconvulsive therapy in patients with schizophrenia: machine learning analysis of resting-state electroencephalography," Schizophrenia Research, vol. 216, pp. 147153, 2020.

[24] R. Redlich, N. Opel, D. Grotegerd et al., "Prediction of individual response to electroconvulsive therapy via machine learning on structural magnetic resonance imaging data," JAMA Psychiatry, vol. 73, no. 6, pp. 557-564, 2016.

[25] A. L. Samuel, "Some studies in machine learning using the game of checkers," IBM Journal of Research and Development, vol. 3, no. 3, pp. 210-229, 1959.

[26] M. A. T. Vu, T. Adal, D. Ba et al., "A shared vision for machine learning in neuroscience," The Journal of Neuroscience, vol. 38, no. 7, pp. 1601-1607, 2018.

[27] M. Khosla, K. Jamison, G. H. Ngo, A. Kuceyeski, and M. R. Sabuncu, "Machine learning in resting-state fMRI analysis," Magnetic Resonance Imaging, vol. 64, pp. 101-121, 2019.

[28] Y. Du, Z. Fu, and V. D. Calhoun, "Classification and prediction of brain disorders using functional connectivity: promising but challenging," Frontiers in Neuroscience, vol. 12, p. 525, 2018.

[29] K. Sakai and K. Yamada, "Machine learning studies on major brain diseases: 5-year trends of 2014-2018," Japanese Journal of Radiology, vol. 37, no. 1, pp. 34-72, 2019.

[30] G. Orrù, W. Pettersson-Yeo, A. F. Marquand, G. Sartori, and A. Mechelli, "Using support vector machine to identify imaging biomarkers of neurological and psychiatric disease: a critical review," Neuroscience and Biobehavioral Reviews, vol. 36, no. 4, pp. 1140-1152, 2012.

[31] B. Sundermann, D. Herr, W. Schwindt, and B. Pfleiderer, "Multivariate classification of blood oxygen level-dependent FMRI data with diagnostic intention: a clinical perspective," AJNR. American Journal of Neuroradiology, vol. 35, no. 5, pp. 848-855, 2014.

[32] D. Librenza-Garcia, B. J. Kotzian, J. Yang et al., "The impact of machine learning techniques in the study of bipolar disorder: a systematic review," Neuroscience and Biobehavioral Reviews, vol. 80, pp. 538-554, 2017.

[33] J. M. Mateos-Pérez, M. Dadar, M. Lacalle-Aurioles, Y. IturriaMedina, Y. Zeighami, and A. C. Evans, "Structural neuroimaging as clinical predictor: a review of machine learning applications," NeuroImage: Clinical, vol. 20, pp. 506-522, 2018.

[34] F. Pereira, T. Mitchell, and M. Botvinick, "Machine learning classifiers and fMRI: a tutorial overview," Neuro Image., vol. 45, 1 Supplement, pp. S199-S209, 2009.

[35] S. Song, Z. Zhan, Z. Long, J. Zhang, and L. Yao, "Comparative study of SVM methods combined with voxel selection for object category classification on fMRI data," PLoS One, vol. 6, no. 2, article e17191, 2011.

[36] H. Zhuang, R. Liu, C. Wu et al., "Multimodal classification of drug-naïve first-episode schizophrenia combining anatomical, diffusion and resting state functional resonance imaging," Neuroscience Letters, vol. 705, pp. 87-93, 2019.

[37] Z. Long, B. Jing, H. Yan et al., "A support vector machinebased method to identify mild cognitive impairment with multi-level characteristics of magnetic resonance imaging," Neuroscience, vol. 331, pp. 169-176, 2016.

[38] J. W. Wu, S. S. Hseu, J. L. Fuh et al., "Factors predicting response to the first epidural blood patch in spontaneous intracranial hypotension," Brain, vol. 140, no. 2, pp. 344352, 2017.

[39] S. Liang, Y. Li, Z. Zhang et al., "Classification of first-episode schizophrenia using multimodal brain features: a combined structural and diffusion imaging study," Schizophrenia Bulletin, vol. 45, no. 3, pp. 591-599, 2019.

[40] L. Breiman, "Random forests," Machine Learning, vol. 45, no. 1, pp. 5-32, 2001.

[41] P. K. Douglas, S. Harris, A. Yuille, and M. S. Cohen, "Performance comparison of machine learning algorithms and number of independent components used in fMRI decoding of belief vs. disbelief," Neuro Image., vol. 56, no. 2, pp. 544-553, 2011.

[42] J. G. Lee, S. Jun, Y. W. Cho et al., "Deep learning in medical imaging: general overview," Korean Journal of Radiology, vol. 18, no. 4, pp. 570-584, 2017.

[43] H. C. Kim, P. A. Bandettini, and J. H. Lee, “Deep neural network predicts emotional responses of the human brain from functional magnetic resonance imaging," Neuro Image., vol. 186, pp. 607-627, 2019.

[44] L. He, H. Li, S. K. Holland, W. Yuan, M. Altaye, and N. A. Parikh, "Early prediction of cognitive deficits in very preterm infants using functional connectome data in an artificial neural network framework," NeuroImage: Clinical, vol. 18, pp. 290 297, 2018.

[45] L. Li, W. Qin, L. Bai, and J. Tian, "Exploring vision-related acupuncture point specificity with multivoxel pattern analysis," Magnetic Resonance Imaging, vol. 28, no. 3, pp. 380-387, 2010.

[46] T. Xue, L. Bai, S. Chen et al., "Neural specificity of acupuncture stimulation from support vector machine classification analysis," Magnetic Resonance Imaging, vol. 29, no. 7, pp. 943-950, 2011.

[47] W.-M. Jung, I.-S. Lee, Y.-S. Lee et al., "Decoding spatial location of perceived pain to acupuncture needle using multivoxel pattern analysis," Molecular Pain, vol. 15, p. 174480691987706 , 2019.

[48] M. M. López, J. M. Górriz, J. Ramírez, M. Gómez-Río, J. Verdejo, and J. Vas, "Component-based technique for determining the effects of acupuncture for fighting migraine using SPECT images," Expert Systems with Applications, vol. 40, no. 1, pp. 44-51, 2013.

[49] H. Yu, X. Li, X. Lei, and J. Wang, "Modulation effect of acupuncture on functional brain networks and classification of its manipulation with EEG signals," IEEE Transactions on Neural Systems and Rehabilitation Engineering, vol. 27, no. 10, pp. 1973-1984, 2019.

[50] J. Liu, J. Mu, T. Chen, M. Zhang, and J. Tian, "White matter tract microstructure of the $\mathrm{mPFC}$-amygdala predicts interindividual differences in placebo response related to treatment in migraine patients," Human Brain Mapping, vol. 40, no. 1, pp. 284-292, 2018.

[51] X.-J. Yang, L. Liu, Z.-L. Xu et al., "Baseline brain gray matter volume as a predictor of acupuncture outcome in treating migraine," Frontiers in Neurology, vol. 11, p. 111, 2020. 
[52] Y. Tao, S. Ruirui, H. Zhaoxuan, M. Peihong, and Z. Fang, "Resting-state functional brain network predicts responses to acupuncture treatment in functional dyspepsia," China Journal of Traditional Chinese Medicine and Pharmacy, vol. 35, no. 5, pp. 2581-2584, 2020.

[53] Y. Tu, A. Ortiz, R. L. Gollub et al., "Multivariate resting-state functional connectivity predicts responses to real and sham acupuncture treatment in chronic low back pain," NeuroImage: Clinical, vol. 23, article 101885, 2019.

[54] W.-s. Hao, X. Zhu, X.-r. Wang, H.-y. Yang, Z.-h. Wang, and Y.-j. Zheng, "Biochemical index variation prediction during electro-acupuncture analgesia using ANFIS method," Journal of Shanghai Jiaotong University, vol. 42, no. 2, pp. 177-180, 2008.

[55] J. J. Xing, B. Y. Zeng, J. Li, Y. Zhuang, and F. R. Liang, “Acupuncture point specificity," International Review of Neurobiology, vol. 111, pp. 49-65, 2013.

[56] G. Chen, N. E. Adleman, Z. S. Saad, E. Leibenluft, and R. W. Cox, "Applications of multivariate modeling to neuroimaging group analysis: a comprehensive alternative to univariate general linear model," Neuro Image., vol. 99, pp. 571-588, 2014.

[57] K. K. S. Hui, J. Liu, N. Makris et al., "Acupuncture modulates the limbic system and subcortical gray structures of the human brain: evidence from fMRI studies in normal subjects," Human Brain Mapping, vol. 9, no. 1, pp. 13-25, 2000.

[58] P. J. Rong, J. J. Zhao, J. H. Gao et al., "Progress of research on specificity of meridian acupoint efficacy," Chinese Journal of Integrative Medicine, vol. 19, no. 12, pp. 889-893, 2013.

[59] L. Bai, X. Niu, Z. Liu et al., "The role of insula-cerebellum connection underlying aversive regulation with acupuncture," Molecular Pain, vol. 14, 2018.

[60] Z. G. Li, M. M. Wu, and C. Z. Liu, "Progress of researches on acupuncture manipulation and its quantification," Acupuncture Research, vol. 35, no. 1, pp. 78-81, 2010.

[61] R. B. Rutledge, A. M. Chekroud, and Q. J. M. Huys, "Machine learning and big data in psychiatry: toward clinical applications," Current Opinion in Neurobiology, vol. 55, pp. 152159, 2019.

[62] F. Zeng, W. Qin, T. Ma et al., "Influence of acupuncture treatment on cerebral activity in functional dyspepsia patients and its relationship with efficacy," The American Journal of Gastroenterology, vol. 107, no. 8, pp. 1236-1247, 2012.

[63] R. Sun, Z. He, P. Ma et al., "The participation of basolateral amygdala in the efficacy of acupuncture with deqi treating for functional dyspepsia," Brain Imaging and Behavior, 2020.

[64] W. Huang, D. Pach, V. Napadow et al., "Characterizing acupuncture stimuli using brain imaging with FMRI-a systematic review and meta-analysis of the literature," PLoS One, vol. 7, no. 4, article e32960, 2012.

[65] C. W. Woo, L. J. Chang, M. A. Lindquist, and T. D. Wager, "Building better biomarkers: brain models in translational neuroimaging," Nature Neuroscience, vol. 20, no. 3, pp. 365377, 2017.

[66] T. Wolfers, J. K. Buitelaar, C. F. Beckmann, B. Franke, and A. F. Marquand, "From estimating activation locality to predicting disorder: a review of pattern recognition for neuroimaging-based psychiatric diagnostics," Neuroscience and Biobehavioral Reviews, vol. 57, pp. 328-349, 2015.

[67] R. A. Poldrack, G. Huckins, and G. Varoquaux, "Establishment of best practices for evidence for Prediction," JAMA Psychiatry, vol. 77, no. 5, p. 534, 2020.
[68] G. Varoquaux, "Cross-validation failure: small sample sizes lead to large error bars," Neuro Image, vol. 180, Part A, pp. 68-77, 2018.

[69] D. Scheinost, S. Noble, C. Horien et al., “Ten simple rules for predictive modeling of individual differences in neuroimaging," Neuro Image., vol. 193, pp. 35-45, 2019.

[70] J. Lee, I. Mawla, J. Kim et al., "Machine learning-based prediction of clinical pain using multimodal neuroimaging and autonomic metrics," Pain, vol. 160, no. 3, pp. 550-560, 2019.

[71] A. A. Nicholson, M. Densmore, M. C. McKinnon et al., "Machine learning multivariate pattern analysis predicts classification of posttraumatic stress disorder and its dissociative subtype: a multimodal neuroimaging approach," Psychological Medicine, vol. 49, no. 12, pp. 2049-2059, 2019.

[72] X. Yang, X. Hu, W. Tang et al., "Multivariate classification of drug-naive obsessive-compulsive disorder patients and healthy controls by applying an SVM to resting-state functional MRI data," BMC Psychiatry, vol. 19, no. 1, p. 210, 2019.

[73] F. Mokhtari, W. J. Rejeski, Y. Zhu et al., "Dynamic fMRI networks predict success in a behavioral weight loss program among older adults," Neuro Image., vol. 173, pp. 421-433, 2018.

[74] D. Wiesen, C. Sperber, G. Yourganov, C. Rorden, and H.O. Karnath, "Using machine learning-based lesion behavior mapping to identify anatomical networks of cognitive dysfunction: spatial neglect and attention," Neuro Image., vol. 201, article 116000, 2019.

[75] D. B. Dwyer, P. Falkai, and N. Koutsouleris, "Machine learning approaches for clinical psychology and psychiatry," Annual Review of Clinical Psychology, vol. 14, no. 1, pp. 91-118, 2018.

[76] K. M. Han, D. De Berardis, M. Fornaro, and Y. K. Kim, "Differentiating between bipolar and unipolar depression in functional and structural MRI studies," Progress in NeuroPsychopharmacology and Biological Psychiatry, vol. 91, pp. 20-27, 2019.

[77] B. Cao, Q. Luo, Y. Fu et al., "Predicting individual responses to the electroconvulsive therapy with hippocampal subfield volumes in major depression disorder," Scientific Reports, vol. 8, no. 1, article 5434, 2018.

[78] J. Young, M. Modat, M. J. Cardoso et al., "Accurate multimodal probabilistic prediction of conversion to Alzheimer's disease in patients with mild cognitive impairment," NeuroImage: Clinical, vol. 2, pp. 735-745, 2013. 